\title{
Moments of continuous bi-variate distributions: An alternative approach
}

\author{
Oyeka ICA ${ }^{1}$, Okeh $\mathrm{UM}^{2}$ \\ ${ }^{1}$ Department of Statistics, Nnamdi Azikiwe University Awka, Nigeria \\ ${ }^{2}$ Department of Industrial Mathematics and Applied Statistics, Ebonyi State University Abakaliki, Nigeria
}

\section{Email address:}

uzomaokey@ymail.com(Okeh UM)

\section{To cite this article:}

Oyeka ICA, Okeh UM. Moments of Continuous Bi-Variate Distributions: An Alternative Approach. Science Journal of Applied Mathematics and Statistics. Vol. x, No. x, 2013, pp. 62-69. doi: 10.11648/j.sjams.20130105.15

\begin{abstract}
We propose a method of obtaining the moment of some continuous bi-variate distributions with parameters $\alpha_{1}, \beta_{1}$ and $\alpha_{2}, \beta_{2}$ in finding the nth moment of the variable $x^{c} y^{d}(c \geq 0, d \geq 0)$ where $\mathrm{X}$ and $\mathrm{Y}$ are continuous random variables having the joint pdf, $\mathrm{f}(\mathrm{x}, \mathrm{y})$.Here we find the so called $g_{n}(c, d)$ defined $g_{n}(c, d)=E\left(X^{c} Y^{d}+\lambda\right)^{n}$, the nth moment of expected value of the $\mathrm{t}$ distribution of the cth power of $\mathrm{X}$ and $\mathrm{dth}$ power of $\mathrm{Y}$ about the constant $\lambda$. These moments are obtained by the use of bi-variate moment generating functions, when they exist. The proposed $g_{n}(c, d)$ is illustrated with some continuous bi-variate distributions and is shown to be easy to use even when the powers of the random variables being considered are non-negative real numbers that need not be integers. The results obtained using $g_{n}(c, d)$ are the same as results obtained using other methods such as moment generating functions when they exist.
\end{abstract}

Keywords: Moment Generating Functions, Bivariate Distributions, Continuous Random Variables, Joint Pdf

\section{Introduction}

Sometimes a researcher's interest may be in finding the nth moment of the variable $x^{c} y^{d}(c \geq 0, d \geq 0)$ where $\mathrm{X}$ and $\mathrm{Y}$ are continuous random variables having the joint pdf, $\mathrm{f}(\mathrm{x}, \mathrm{y})$ (Baisnab and Manoranjan,

1993;Freund,1992;Uche, 2003). The moment is to be taken about some constant $\lambda$ (Hay,1973). The method that has generally recommended itself here is the use of bi-variate moment generating functions, when they exist to obtain these moments. However, moment generating functions can sometimes be difficult to repeatedly differentiate and evaluate especially when $n$ becomes large(Hay,1973;Spiegel,1998).

We here propose the so called $g_{n}(c, d)$ defined $g_{n}(c, d)=E\left(X^{c} Y^{d}+\lambda\right)^{n}$, the nth moment of expected value of the $t$ distribution of the cth power of $X$ and $d$ th power of $Y$ about the constant $\lambda$. That is,

$$
g_{n}(c, d)=E\left(x^{c} y^{d}+\lambda\right)^{n}
$$

Now,

$$
\begin{aligned}
& g_{n}(c, d)=E\left(x^{c} y^{d}+\lambda\right)^{n}=\int_{-\infty}^{\infty} \int_{-\infty}^{\infty}\left(x^{c} y^{d}+\lambda\right)^{n} f(x, y) d x d y \\
& =\int_{-\infty}^{\infty} \int_{-\infty}^{\infty} \sum_{t=0}^{n}\left(\begin{array}{l}
n \\
t
\end{array}\right) \lambda^{n-t} x^{c t} y^{d t} f(x, y) d x d y \\
& \therefore g_{n}(c, d)=\sum_{t=0}^{n}\left(\begin{array}{l}
n \\
t
\end{array}\right) \lambda^{n-t} \int_{-\infty}^{\infty} \int_{-\infty}^{\infty} x^{c t} y^{d t} f(x, y) d x d y \\
& =\sum_{t=0}^{n}\left(\begin{array}{l}
n \\
t
\end{array}\right) \lambda^{n-t} \mu_{c t, d t}^{\prime} \\
& \text { where } \mu_{c t, d t}^{\prime}=\int_{-\infty}^{\infty} \int_{-\infty}^{\infty} x^{c t} y^{d t} f(x, y) d x d y
\end{aligned}
$$

is the $\mathrm{t}^{\text {th }}$ moment of the joint distribution of $X^{c}$ and $Y^{d}$ about zero.

Note: that as expected $g_{0}^{(0,0)}=1$.

Also,

$$
g_{1}^{(1,1)}=E(x, y)+\lambda=\mu_{x y}^{\prime}+\lambda
$$

Where $\mu_{x y}^{\prime}$ is the mean of joint probability distribution of $\mathrm{X}$ and $\mathrm{Y}$ about zero 


$$
g_{1}^{(1 ; 1)}=0 \text { when } \lambda=-\mu_{x y}
$$

The variance of joint distribution of $\mathrm{X}$ and $\mathrm{Y}$ is given by

$$
p_{0_{x, y}}^{2}=g_{2}^{(1 ; 1)}-g_{1}^{(1 ; 1)^{2}}=g_{2}^{(1 ; 1)}\left(\text { if } \lambda=-\mu_{x, y}\right)
$$

It is easily seen from equations 1 and 2 that,

$$
{ }_{x} g_{n}^{(c)}=g_{n}^{(c, 0)} \text { and }{ }_{y} g_{n}^{(d)}=g_{n}^{(0, d)}
$$

\subsection{To Illustrate}

Suppose $\mathrm{X}$ and $\mathrm{Y}$ have bi-variate exponential distribution with parameters $\beta_{1}$ and $\beta_{2}$ that is

$$
\mathrm{f}(\mathrm{x}, \mathrm{y})=\frac{1}{\beta_{1} \beta_{2}} \ell^{-\left(\frac{x}{\beta_{1}}+\frac{y}{\beta_{y}}\right)}
$$

Then

$$
\begin{aligned}
& g_{n}^{(c ; d)}=E\left(X^{c} Y^{d}+\lambda\right)^{n} \\
& =\sum_{t=0}^{n}\left(\begin{array}{c}
n \\
t
\end{array}\right) \lambda^{n-t} \int_{0}^{\infty} \int_{0}^{\infty} x^{c t} y^{d t} f(x, y) d x d y \\
& =\frac{1}{\beta_{1} \beta_{2}} \sum_{t=0}^{n}\left(\begin{array}{c}
n \\
t
\end{array}\right) \lambda^{n-t} \int_{0}^{\infty} \int_{0}^{\infty} x^{c t} y^{d t} e^{-\left(\frac{x}{\beta_{1}}+\frac{y}{\beta_{2}}\right)} d x d y \\
& \left.\left.g_{n}^{(c ; d ; \lambda)}=\sum_{t=0}^{n}\left(\begin{array}{l}
n \\
t
\end{array}\right) \lambda^{n-t} \beta_{1}^{c t} \beta_{2}^{d t}\right) c t+1\right) d t+1
\end{aligned}
$$

For instance

$$
g _ { 2 } ^ { ( 2 ; 3 ; \lambda ) } = \sum _ { t = 0 } ^ { 2 } ( \begin{array} { l } 
{ 2 } \\
{ t }
\end{array} ) \lambda ^ { 2 - t } \beta _ { 1 } ^ { 2 t } \beta _ { 2 } ^ { 3 t } \sqrt [ 2 t ] { 2 } + 1 \longdiv { 3 t } + 1
$$

For $\mathrm{t}=0,1,2$ for example for $\mathrm{t}=0$, we have

$$
\left(\begin{array}{l}
2 \\
0
\end{array}\right) \lambda^{2-0} \times 1=\lambda^{2}
$$

For $\mathrm{t}=1$;

$$
\left(\begin{array}{l}
2 \\
1
\end{array}\right) \lambda^{2-1} \beta_{1}^{2} \times \beta_{2}^{3} \times \sqrt{3} \times \sqrt{4}=2 \lambda \beta_{1}^{2} \times \beta_{3}^{2} \times 2 \times 6=24 \lambda \beta_{1}^{2} \beta_{2}^{3}
$$

For $\mathrm{t}=2$;

$$
\begin{gathered}
\left(\begin{array}{l}
2 \\
2
\end{array}\right) \lambda^{0} \beta_{1}^{4} \times \beta_{2}^{6} \times \sqrt{5} \times \sqrt{7}=\beta_{1}^{4} \times \beta_{2}^{6} \times(4 ! 6 !)=17,280 \beta_{1}^{4} \beta_{2}^{6} \\
g_{2}^{(2 ; 3 ; \lambda)}=\lambda^{2}+24 \lambda \beta_{1}^{2} \beta_{2}^{3}+17,280 \beta_{1}^{4} \beta_{2}^{6}
\end{gathered}
$$

If $\mathrm{X}$ and $\mathrm{Y}$ have bi-variate exponential distribution with parameters $\beta_{1} \beta_{2}$, so that

$$
f(x, y)=\frac{1}{\beta_{1} \beta_{2}} e^{-\left(\frac{x}{\beta_{1}}+\frac{y}{\beta_{y}}\right)} ; x>0, y>0
$$

Then

$$
\begin{aligned}
& \left.\left.g_{3}^{(1 ; 1 ; \lambda)}=\sum_{t=0}^{n}\left(\begin{array}{l}
3 \\
t
\end{array}\right) \lambda^{3-t} \beta_{1}^{t} \beta_{2}^{t}\right) t+1\right) t \\
& =3 \mu_{0} \lambda^{3}+\left(\begin{array}{l}
3 \\
1
\end{array}\right) \times \lambda^{2} \times \beta_{1} \times \beta_{2}+\left(\begin{array}{l}
3 \\
2
\end{array}\right) \times \\
& \times \lambda^{1} \times \beta_{1}{ }^{2} \times \beta_{2}^{2} \times 4+\left(\begin{array}{l}
3 \\
3
\end{array}\right) \times \beta_{1}^{3} \times \beta_{2}^{3} \times 36 . \\
& =\lambda^{3}+3 \lambda^{2} \beta_{1} \beta_{2}+12 \lambda \beta_{1}^{2} \beta_{2}^{2}+36 \beta_{1}^{3} \beta_{2}^{3} \\
& \text { If } \lambda=-\beta_{1} \beta_{2} \text { then }, \\
& g_{3}^{\left(1 ; 1 ;-\beta_{1} \beta_{2}\right)}=-\beta_{1}^{3} \beta_{2}^{3}+3 \beta_{1}^{3} \beta_{2}^{3}-12 \beta_{1}^{3} \beta_{2}^{3}+36 \beta_{1}^{3} \beta_{2}^{3} \\
& =26 \beta_{1}^{3} \beta_{2}^{3}
\end{aligned}
$$

This result is the third moment about the mean of the joint exponential distribution of $\mathrm{X}$ and $\mathrm{Y}$ with parameters $\beta_{1}$ and $\beta_{2}$.Note that using the familiar moment generating function approach to obtain this type of moment would strictly speaking require one to first find the joint 'mgf' of the two random variables about there means; $\mu_{1}$ and $\mu_{2}$ before carrying out the necessary differentiations. That is one would have to find

$$
M_{X-\beta_{1}, Y-\beta_{2}}{ }^{\left(t_{1}, t_{2}\right)}=e^{-\left(\beta_{1} t_{1}+\beta_{2} t_{2}\right)} \times M_{X, Y}{ }^{\left(t_{1}, t_{2}\right)}
$$

Where $M_{X, Y}{ }^{\left(t_{1}, t_{2}\right)}$ is the joint mgf of X and Y for the present example. We would have to first differentiate $e^{-\left(\beta_{1} t_{1}+\beta_{2} t_{2}\right)\left(1-\beta_{1} t_{1}\right)^{-1}\left(1-\beta_{2} t_{2}\right)^{-1}}$ three times and evaluate the results at $\mathrm{t}_{1}=0$ and $\mathrm{t}_{2}=0$, yielding the value $26 \beta_{1}^{3} \beta_{2}^{3}$, the same result that was more easily obtained using the proposed $g_{n}^{(c ; d)}=g_{3}^{(1 ; 1)}$ here. If interest is in finding the Skewness (Sk), kurtosis (Ku) of the joint distribution of $\mathrm{X}$ and Y. This can be obtained from the expressions;

$$
\begin{aligned}
& S K=\frac{g_{3}^{(1 ; 1)}}{\left(g_{2}^{(1,1)}\right)^{\frac{3}{2}}}\left(\lambda=-\mu_{x y}\right) \\
& \text { and } \\
& K U=\frac{g_{4}^{(1 ; 1)}}{\left(g_{2}^{(1,1)}\right)^{2}}\left(\lambda=-\mu_{x y}\right)
\end{aligned}
$$

For the Bi-variate Gamma distribution with $\alpha_{1}=\alpha_{2}=1$ and,

$$
f(x, y)=\frac{1}{\beta_{1} \beta_{2}\left(\beta_{1}+\beta_{2}\right)(y+x)} e^{-\left(\frac{x}{\beta_{1}}+\frac{y}{\beta_{2}}\right)}, x>0, y>0
$$

Thus $g_{n}^{(c, d)}$ can be easily obtained using equation 2 as

$$
g_{n}^{(c, d)}=\frac{\left.\left.\left.\left.\left.\sum\left(\begin{array}{l}
n \\
t
\end{array}\right) \lambda^{n-t} \beta_{1}^{t} \beta_{2}^{u t}(\beta) \sqrt{(c t}+2\right)\right)(d t+1)+\beta_{2}\right)(c t+1)\right)(d t+2)\right)}{\beta+\beta_{2}}
$$

It is easy to calculate from equation 12 that the mean and variance of the joint distribution in equation 11 are from equations 4 and $5,2 \beta_{1} \beta_{2}$ and $8 \beta_{1}^{2} \beta_{2}^{2}$ respectively. The 
third moment about the mean of distribution is $88 \beta_{1}^{3} \beta_{2}^{3}$ while the fourth moment is $2016 \beta_{1}^{4} \beta_{2}^{4}$

Evaluating

$$
\begin{aligned}
& \lambda^{4}+4 \lambda^{3}=16 \beta_{1}{ }^{4} \beta_{2}^{4} \\
& \lambda^{4}+4 \lambda^{3}\left(2 \beta_{1} \beta_{2}\right)=-16 \beta_{1}{ }^{4} \beta_{2}{ }^{4} \\
& +6 \lambda^{2}\left(12 \beta_{1}{ }^{2} \beta_{2}{ }^{2}\right)=+288 \beta_{1}{ }^{4} \beta_{2}{ }^{4} \\
& +4 \lambda\left(144 \beta_{1}{ }^{3} \beta_{2}^{3}\right)=-1152 \beta_{1}{ }^{3} \beta_{2}^{4} \\
& =2880 \beta_{1}^{4} \beta_{2}{ }^{4}=+2880 \beta_{1}^{4} \beta_{2}^{4}
\end{aligned}
$$

The moment generating function for Equation 11 is

$$
\frac{\beta_{1}+\beta_{2}-\beta_{1} \beta_{2}\left(t_{1}+t_{2}\right)}{\left(\beta_{1}+\beta_{2}\right)\left(1-\beta_{1} t_{1}\right)^{2}\left(1-\beta_{2} t_{2}\right)^{2}}
$$

Which is clearly difficult to use in obtaining the above moments than using the proposed $g_{n}^{(c, d)}$.

Note that the moments of distribution of $\mathrm{X}$ can be easily obtained by evaluating $g_{x n}^{(c)}$ got from equation 12 as

$$
g_{x n}^{(c)}=g_{n}^{(c ; 0)}=\frac{\left.\left.\sum\left(\begin{array}{l}
n \\
t
\end{array}\right) \lambda^{n-t} \beta_{1}^{c t}(\beta) \sqrt{c t}+2+\beta_{2}\right) c t+1\right)}{\beta_{1}+\beta_{2}}
$$

Similarly for $g_{y}^{(n ; d)} \rightarrow g_{y}^{n(d)}$

$$
g_{y}^{(c ; d)}=g_{n}^{(0 ; d)}=\frac{\left.\left.\sum\left(\begin{array}{l}
n \\
t
\end{array}\right) \lambda^{n-t} \beta_{2}^{d t}\left(\beta_{1}\right) d t+1+\beta_{2}\right) d t+2\right)}{\beta_{1}+\beta_{2}}
$$

These are also easier and faster to use in evaluating the moments of the distributions of $\mathrm{X}$ and $\mathrm{Y}$ respectively of equation 11 then using the corresponding moment generating functions.

\section{Bi-Variate MomenT Generating Function}

$$
\begin{aligned}
& g_{n}^{(c ; d)}=E\left(\lambda_{1} X^{c}+\lambda_{2} Y^{d}\right)^{n} \\
& =\iint\left(\lambda_{1} X^{c}+\lambda_{2} Y^{d}\right)^{n} f(X, Y) d y d x \\
& =\iint \sum_{t=0}^{n}\left(\begin{array}{l}
n \\
t
\end{array}\right) \lambda_{1}^{t} \lambda_{2}^{n-t} x^{c t} y^{(n-t) d} f(x, y) d y d x \\
& =\sum_{t=0}^{n}\left(\begin{array}{l}
n \\
t
\end{array}\right) \lambda_{1}^{t} \lambda_{2}^{n-t} \iint X^{c t} Y^{(n-t) d} f(x, y) d y d x \\
& =\sum_{t=0-n}^{n}\left(\begin{array}{l}
n \\
t
\end{array}\right) \lambda_{1}^{t} \lambda_{2}^{n-t} \mu_{c t,(n-t) d}^{\prime} \\
& \text { where } \mu_{c t,(n-t) \times d}^{\prime}=\iint x^{c t} y^{(n-t) d} f(x, y) d y d x
\end{aligned}
$$

Is the joint cth and $(n-t) \times d$ th moment of the distribution of $\mathrm{X}, \mathrm{Y}$ about the origin.

Note that

$$
\begin{aligned}
& g_{1}^{(1 ; 1)}=E\left(\lambda_{1} X+\lambda_{2} Y\right)^{1} \\
& =\lambda_{1} \mu_{1}+\lambda_{2} \mu_{2} \text {. } \\
& \text { if } \lambda_{1}=\lambda_{2}=1 \text {. } \\
& \text { the } n g_{1}^{(1 ; 1)}=\mu_{1}+\mu_{2} \text {. } \\
& \text { if } d=0 \text { and } \lambda_{1}=1 \text {, } \\
& w e h a v e \text { that } \\
& g_{n}^{(c ; 0)}=g_{n c} a n d \text { if } c=0 \text {, } \\
& a n d \quad \lambda_{2}=1 \\
& \text { the } n \\
& g_{n}^{(0 ; d)}=g_{n}^{(d)}
\end{aligned}
$$

Note also that $g_{n}^{(0 ; 0)}=1$.

Example.

Let

$$
\begin{aligned}
& f(x, y)=\beta_{1}^{2} \beta_{2}^{2} x y e^{-\left(\beta_{1} x \beta_{2} y\right)}, x>0, y>o \\
& \text { then } \\
& g_{n}^{(c ; d)}=E\left(\lambda_{1} X^{c}+\lambda_{2} Y^{d}\right)^{n} \\
& =\sum_{t=0-n}^{n}\left(\begin{array}{l}
n \\
t
\end{array}\right) \lambda_{1} \lambda_{2}^{n-t} \iint X^{c t} Y^{(n-t) d} f(x, y) \\
& \text { then } \\
& \beta_{1}^{2} \beta_{2}^{2} x y e^{-\left(\beta_{1} x \beta_{2} y\right)} d x d y \\
& =\beta_{1}^{2} \beta_{2}^{2} \sum_{t=0-n}^{\left(\begin{array}{l}
n \\
t
\end{array}\right)} \lambda_{1}^{t} \lambda_{2}^{n-t} \iint X^{c t+1} Y^{(n-t) d+1} e^{-\left(\beta_{1} x+\beta_{2} y\right)} d x d y \\
& \left.\left.=\sum\left(\begin{array}{l}
n \\
t
\end{array}\right) \lambda_{1}^{t} \lambda_{2}^{n-t} \beta_{1}^{-c t} \beta_{2}^{-(n-t) d}\right) c t+2+\beta_{2} \sqrt{(n}-t\right)^{d+2}
\end{aligned}
$$

Finding

$$
\begin{aligned}
& g_{1}^{(1 ; 1)}=E\left(\lambda_{1} X+\lambda_{2} Y\right)^{1} \\
& \left.\left.=\sum\left(\begin{array}{l}
1 \\
t
\end{array}\right) \lambda_{1}^{t} \lambda_{2}^{1-t} \beta_{1}^{-t} \beta_{2}^{-(1-t)^{1}}\right) t+2+\sqrt{(1}-t\right)^{+2}
\end{aligned}
$$

Evaluating for $\mathrm{t}=0$

$$
\begin{aligned}
& \left.( \begin{array} { l } 
{ 1 } \\
{ 0 }
\end{array} ) \lambda _ { 1 } ^ { 0 } \lambda _ { 2 } ^ { 1 } \beta _ { 1 } ^ { 1 } \beta _ { 2 } ^ { - 1 } \longdiv { 2 }\right) 3 \\
& =\text { (1) } \lambda _ { 1 } { } _ { 1 } \lambda _ { 2 } { } ^ { 2 } \beta _ { 1 } { } _ { 1 } ^ { 1 } \beta _ { 2 } { } ^ { - 1 } \sqrt { 1 } \longdiv { 2 } = \frac { 2 \lambda _ { 2 } } { \beta _ { 2 } }
\end{aligned}
$$

Evaluating for $\mathrm{t}=1$

$$
\begin{aligned}
& \left(\begin{array}{l}
1 \\
0
\end{array}\right) \lambda_{1}^{1} \lambda_{2}^{0} \beta_{1}^{-1} \beta_{2}^{-0} \sqrt{3} \sqrt{2}=\sqrt{1} \times \beta_{1}^{-1} \times 2=\frac{2 \lambda_{1}}{\beta_{1}} \\
& \therefore \quad g_{1}^{(1 ; 1)}=\frac{2 \lambda_{1}}{\beta_{1}}+\frac{2 \lambda_{2}}{\beta_{2}}
\end{aligned}
$$

Suppose $\mathrm{c}=1, \mathrm{~d}=1$ and $\mathrm{n}=2$

Evaluating for $\mathrm{t}=2$ 


$$
\begin{aligned}
& g_{2}^{(1 ; 1)}=E\left(\lambda_{1} X+\lambda_{2} Y\right)^{2}= \\
& \left.\left.=\sum_{t=0}^{2}\left(\begin{array}{l}
2 \\
t
\end{array}\right) \lambda_{1}^{t} \lambda_{2}^{2-t} \beta_{1}^{-t} \beta_{2}^{-(2-t) d=1}\right) t+2\right)(2-t)+2
\end{aligned}
$$$$
\text { For } t=0
$$$$
\left(\begin{array}{l}
2 \\
0
\end{array}\right) \lambda_{1}^{0} \lambda_{2}^{2} \beta_{1}^{-0} \beta_{2}^{-(2-0)} \sqrt{2} \overline{4}=\lambda_{2}^{2} \times \frac{6 \lambda_{2}^{2}}{\beta_{2}^{2}}
$$$$
\text { For } t=1
$$$$
\left(\begin{array}{l}
2 \\
1
\end{array}\right) \lambda_{1}^{1} \lambda_{2}^{1} \beta_{1}^{-1} \beta_{2}^{-1} \sqrt{3} \sqrt{3}=\frac{6 \lambda_{1} \lambda_{2}}{\beta_{1} \beta_{2}}
$$$$
\text { For } t=2
$$$$
\left(\begin{array}{l}
2 \\
2
\end{array}\right) \lambda_{1}^{1} \lambda_{2}^{0} \beta_{1}^{-2} \beta_{2}^{0} \sqrt{4} \sqrt{2}=\lambda_{1}^{2} \times \frac{6}{\beta_{1}^{2}}=\frac{6 \lambda_{1}^{2}}{\beta_{1}^{2}}
$$$$
\therefore g_{2}^{(1-1)}=\frac{6 \lambda_{1}^{2}}{\beta_{1}^{2}}+\frac{6 \lambda_{1} \lambda_{2}}{\beta_{1} \beta_{2}}+\frac{6 \lambda_{2}^{2}}{\beta_{2}^{2}}
$$

The variance of the joint distribution of $\mathrm{X}, \mathrm{Y}$

$$
\begin{aligned}
& g_{n}^{(c ; d)}=E\left(X^{c} Y^{d}+\lambda\right)^{n} \\
& =\sum_{t=0}^{n}\left(\begin{array}{l}
n \\
t
\end{array}\right) \lambda^{n-t} \int_{0}^{\infty} \int_{0}^{\infty} x^{c t} y^{d t} f(x, y) d x d y \\
& =\frac{1}{\beta_{1} \beta_{2}} \sum_{t=0}^{n}\left(\begin{array}{l}
n \\
t
\end{array}\right) \lambda^{n-t} \int_{0}^{\infty} \int_{0}^{\infty} x^{c t} y^{d t} e^{-\left(\frac{x}{\beta_{1}}+\frac{y}{\beta_{2}}\right)} d x d y \\
& g_{y}^{(n ; d)} \rightarrow g_{y}^{n(d)} \\
& g_{2}^{(1-1)}-g_{1}^{(1-1)^{2}} \\
& =\frac{6 \lambda_{1} \lambda_{2}}{\beta_{1} \beta_{2}}+\frac{6 \lambda_{2}^{2}}{\beta_{2}^{2}}+\frac{6 \lambda_{1}^{2}}{\beta_{1}^{2}}-\left(\frac{2) 1}{\beta_{1}}+\frac{2 \sqrt{2}}{\beta_{2}}\right)^{2} \\
& =\frac{6 \lambda_{1}^{2}}{\beta_{1}^{2}}+\frac{6 \lambda_{1} \lambda_{2}}{\beta_{1} \beta_{2}}+\frac{6 \lambda_{2}^{2}}{\beta_{1}^{2}}-\left(\frac{4 \lambda_{1}^{2}}{\beta_{1}^{2}}+\frac{6 \lambda_{1} \lambda_{2}}{\beta_{1} \beta_{2}}+\frac{4 \lambda_{2}^{2}}{\beta_{2}^{2}}\right) \\
& =\frac{2 \sqrt{1^{2}}}{\beta_{2}^{2}}+\frac{22^{2}}{\beta_{2}^{2}}
\end{aligned}
$$

$g_{n}^{(c)}$ as noted above,

$g_{x n}^{(c)}=g_{n}^{(c ; 0)}$ for when $\lambda_{1}=1$,

$d=0$, In the present example

$$
\begin{aligned}
& g_{x n}^{(c)}=\sum\left(\begin{array}{c}
n \\
t
\end{array}\right) \lambda_{2}^{n-t} \beta_{1}^{-c t} \sqrt{c t}+2 \times \sqrt{2} \\
& \left.=\sum\left(\begin{array}{l}
n \\
t
\end{array}\right) \lambda_{2}^{n-t} \beta_{1}^{-c t}\right) c t+2
\end{aligned}
$$

similarly

$g_{y n}^{(d)}=g_{n}^{(0 ; d)}$ when $\lambda_{2}=1$,

$c=0$. In the present example

$\left.g_{y n}^{(d)}=\sum\left(\begin{array}{l}n \\ t\end{array}\right) \lambda_{1}^{t} \beta_{2}^{-(n-t) d}\right) \sqrt[(n-t)^{d}]{ }+2$.

Note that; with $\lambda_{1}=1$

$$
\begin{aligned}
& g_{x_{1}}^{(1)}=g_{1}^{(1 ; 0)}=\sum\left(\begin{array}{l}
n \\
t
\end{array}\right) \lambda_{2}^{1-t} \beta_{1}^{-t} \overline{(t+2)} \\
& =\left(\begin{array}{l}
1 \\
0
\end{array}\right) \lambda_{2}^{1-0} \beta_{1}^{0} \overline{2}+\left(\begin{array}{l}
1 \\
1
\end{array}\right) \lambda_{2}^{0} \beta_{1}^{-1} \overline{1+2} \\
& =\lambda_{2}+\frac{2}{\beta_{1}}
\end{aligned}
$$

similarly with $\lambda_{2}=1$, we have that $g_{y_{1}}^{(1)}=g_{1}^{(0 ; 1)}$

$\sum\left(\begin{array}{l}n \\ t\end{array}\right) \lambda_{1}^{t} \beta_{2}^{-(1-t)} \overline{(1-t)}+2$

$$
\begin{aligned}
& \left.\left(\begin{array}{l}
1 \\
0
\end{array}\right) \lambda_{1}^{0} \beta_{2}^{-1} \overline{1+2}+\left(\begin{array}{l}
1 \\
1
\end{array}\right) \lambda_{1}^{1} \beta_{2}^{0}\right) 2 \\
& \left.\left(\begin{array}{l}
1 \\
0
\end{array}\right) \lambda_{1}^{0} \beta_{2}^{-1} \overline{1+2}+\left(\begin{array}{l}
1 \\
1
\end{array}\right) \lambda_{1}^{1} \beta_{2}^{0}\right) 2 \\
& =\frac{2}{\beta^{2}+\lambda_{1}}=\lambda_{1}+\frac{2}{\beta^{2}}
\end{aligned}
$$

The $g_{n}^{(c ; d)}$ for the bi-variate exponential distributions with parameters $\beta_{1}, \beta_{2}$ is 


$$
\begin{aligned}
& g_{n}^{(c ; d)}=E\left(\lambda_{1} X^{c}+\lambda_{2} Y^{d}\right)^{n} \\
& =\frac{1}{\beta_{1} \beta_{2}} \sum\left(\begin{array}{l}
n \\
t
\end{array}\right) \lambda_{1}^{t} \lambda_{2}^{n-t} \int_{-\infty}^{\infty} \int_{-\infty}^{\infty} x^{c t} y^{(n-t) d} \ell^{-\left(\frac{x}{\beta_{1}}+\frac{y}{\beta_{2}}\right)} d y d x \\
& =\sum^{\left(\begin{array}{l}
1 \\
t
\end{array}\right)} \lambda_{1}^{t} \lambda_{2}^{n-t} \beta_{1}^{c t} \beta_{2}^{(n-t) d} \sqrt{c t+1} \overline{(n-t) d}+1
\end{aligned}
$$

For example

$g_{2}^{(2 ; 3)}=E\left(\lambda_{1} X^{2} \lambda_{2} Y^{3}\right)^{2}$

$=\sum\left(\begin{array}{l}2 \\ t\end{array}\right) \lambda_{1}^{t} \lambda_{2}^{2-t} \beta_{1}^{2 t} \beta_{2}^{3(2-t)} \sqrt{2 t}+1 \times \longdiv { 3 ( 2 - t ) } + 1$

$\left(\begin{array}{l}2 \\ 0\end{array}\right) \lambda_{1}^{0} \lambda_{2}^{2} \beta_{1}^{0} \beta_{2}^{6} \sqrt{1} \sqrt{7}=\lambda_{2}^{2} \beta_{2}^{6} 6$ !

$=\lambda^{2}+6 \times \beta_{2}^{6}=720 \lambda_{2}^{2} \beta_{2}^{6}$

For $t=1$ : we have;

$( \begin{array} { l } { 2 } \\ { 1 } \end{array} ) \lambda _ { 1 } ^ { 1 } \lambda _ { 2 } ^ { 1 } \beta _ { 1 } ^ { 2 } \beta _ { 2 } ^ { 3 } \sqrt [ 3 ] { 4 } = 2 \lambda _ { 1 } \lambda _ { 2 } \beta _ { 1 } ^ { 2 } \beta _ { 2 } ^ { 3 } \longdiv { 3 } \overline { 4 }$

$=2 \lambda_{1} \lambda_{2} \beta_{1}^{2} \beta_{2}^{3} 12=24 \lambda_{1} \lambda_{2} \beta_{1}^{2} \beta_{2}^{3}$

For $t=2$ : we have;

$\left.=\left(\begin{array}{l}2 \\ 2\end{array}\right) \lambda_{1}^{2} \lambda_{2}^{0} \beta_{1}^{4} \beta_{2}^{0}\right) \overline{4}+1 \times \sqrt{2}=\lambda_{1}^{2} \beta_{1}^{4} 4 !=6 \lambda_{1}^{2} \beta_{1}^{4}$

$g_{2}^{(2 ; 3)}=6 \lambda_{1}^{2} \times \beta_{1}^{4}+24 \lambda_{1} \lambda_{2} \beta_{1}^{2} \beta_{2}^{3}+720 \lambda_{2}^{2} \beta_{2}^{6}$

Similarly:

$$
\begin{aligned}
& g_{1}^{(2 ; 3)}=E\left(\lambda_{1} X^{2} \lambda_{2} Y^{3}\right)^{\prime} \\
& \left.=\sum\left(\begin{array}{l}
1 \\
t
\end{array}\right) \lambda_{1}^{t} \lambda_{2}^{1-t} \beta_{1}^{2 t} \beta_{2}^{3(1-t)} \sqrt[2 t]{ }+1\right) \sqrt[3(1-t)]{ }+1
\end{aligned}
$$

For $t=0$ : we have;

$$
\begin{aligned}
& =\left(\begin{array}{l}
1 \\
0
\end{array}\right) \lambda_{1}^{0} \lambda_{2}^{1} \beta_{1}^{0} \beta_{2}^{3} \overline{1} \times \overline{4} \\
& =\lambda_{2} \beta_{2}^{3} 6=6 \lambda_{2} \beta_{2}^{3}
\end{aligned}
$$

For $t=1$ : we have;

$$
\begin{aligned}
& \left.=\left(\begin{array}{l}
1 \\
1
\end{array}\right) \lambda_{1}^{1} \lambda_{2}^{0} \beta_{1}^{2} \beta_{2}^{0} \sqrt{3} \times\right)=\lambda_{1} \beta_{1}^{2} 2=2 \lambda_{1} \beta_{1}^{2} \\
& \therefore g_{1}^{(2 ; 3)}=2 \lambda_{1} \beta_{1}^{2}+6 \lambda_{2} \beta_{2}^{3} \\
& \therefore g_{1}^{(2 ; 3)^{2}}=\left(2 \lambda_{1} \beta_{2}^{2}+6 \lambda_{2} \beta_{2}^{3}\right)^{2} \\
& =4 \lambda_{1}^{2} \beta_{1}^{4}+24 \lambda_{1} \lambda_{2} \beta_{1}^{2} \beta_{2}^{3}+36 \lambda_{2}^{2} \beta_{2}^{6} \\
& \text { variance }=g_{2}^{(2 ; 3)}-g_{1}^{(2 ; 3)^{2}} \\
& =6 \lambda_{1}^{2} \beta_{1}^{4}+24 \lambda_{1} \lambda_{2} \beta_{1}^{2} \beta_{2}^{3}+720 \lambda_{2}^{2} \beta_{2}^{6} \\
& -4 \lambda_{1}^{2} \beta_{1}^{4}+24 \lambda_{1} \lambda_{2} \beta_{1}^{2} \beta_{2}^{3}-36 \lambda_{2}^{2} \beta_{2}^{6} \\
& =2 \lambda_{1}{ }^{2} \beta_{1}^{4}+684 \lambda_{2}^{2} \beta_{2}^{6}
\end{aligned}
$$

For the bi-variate exponential distributions

$$
\begin{aligned}
& g_{y n}^{(d)}=g_{n}^{(o ; d)} \\
& =\sum_{t=0}^{n}\left(\begin{array}{l}
n \\
t
\end{array}\right) \lambda_{1}^{t} \beta_{2}^{(n-t) d} \sqrt{(n-t) d}+1\left(\lambda_{2}^{0}\right) \\
& \therefore g_{y 2}^{(3)}=\sum_{t=0}^{n}\left(\begin{array}{l}
2 \\
t
\end{array}\right) \lambda_{1}^{t} \beta_{2}^{(2-t) 3} \overline{(3-(2-t)}-1
\end{aligned}
$$

For $t=0$, we have,

$$
\left(\begin{array}{l}
2 \\
0
\end{array}\right) \lambda_{1}^{0} \beta_{2}^{6} \times \sqrt{7}=720 \lambda_{2}^{6}
$$

For $t=1$, we have,

$$
\begin{aligned}
& \left(\begin{array}{l}
2 \\
1
\end{array}\right) \lambda_{1}^{1} \beta_{2}^{3} \times \overline{4}=12 \lambda_{1} \beta_{2}^{3} \\
& F \text { ort }=2, \text { we have } \\
& \left.\left(\begin{array}{l}
2 \\
2
\end{array}\right) \lambda_{1}^{2} \beta_{2}^{0} \times\right) 1=\lambda_{1}^{2} \\
& g_{2}^{(2 ; 3)}=\lambda_{1}^{2}+12 \lambda_{1} \beta_{2}^{3}+720 \beta_{2}^{6}
\end{aligned}
$$

$$
g_{y}^{(1 ; 3)}=\sum\left(\begin{array}{l}
1 \\
t
\end{array}\right) \lambda_{1}^{t} \beta_{2}^{3(1-t)} \sqrt{3(1-t)}+1
$$

For $t=0$, we have

$$
\left(\begin{array}{l}
1 \\
0
\end{array}\right) \lambda_{1}^{0} \beta_{2}^{3} \times \sqrt{4}=6 \beta_{2}^{3}
$$

For $t=1$

\section{Similarly}

$\left(\begin{array}{l}1 \\ 1\end{array}\right) \lambda_{1}^{1} \beta_{2}^{0} \times \longdiv { 1 } = \lambda _ { 1 }$

$\therefore$ finally $g_{y}^{(1 ; 3)}=\lambda_{1}+6 \beta_{2}^{3}$

$\therefore g_{y}^{(1 ; 3)^{2}}=\left(\lambda_{1}+6 \beta_{2}^{3}\right)^{2}=\lambda_{1}^{2}+12 \lambda_{1} \beta_{2}^{3}+36 \beta_{2}^{6}$

finally

$$
\begin{aligned}
& \text { variance } g_{y}^{(2 ; 3)}-g_{y}^{(1 ; 3)^{2}} \\
& =\lambda_{1}^{2}+12 \lambda_{1} \beta_{2}^{3}+720 \beta_{2}^{6}-\lambda_{1}^{2}-12 \lambda_{1} \beta_{2}^{3}-36 \beta_{2}^{6} \\
& =684 \beta_{2}^{6}
\end{aligned}
$$

For the bi-variate Gamma distribution with $\alpha_{1}=\alpha_{2}=1$ and $\quad p d f$ $f(x, y)=\frac{1}{\beta_{1} \beta_{2}}\left(\beta_{1}+\beta_{2}\right)^{(x+y)} e^{-\left(\frac{x}{\beta_{1}}+\frac{y}{\beta_{2}}\right)}, x>0 ; y>0$

Suppose $\mathrm{X}$ and $\mathrm{Y}$ have a joint bivariate Gamma distribution with parameters $\alpha_{1}$ and $\beta_{1}$ and $\alpha_{2}$ and $\beta_{2}$ with pdf 


$$
\begin{aligned}
f(x, y)= & \frac{1}{\beta_{2} \beta_{1}^{\alpha_{1}} \sqrt{\alpha_{1}}+\beta_{1} \beta_{2}^{\alpha_{2}} \sqrt{\alpha_{2}}}\left(x^{\alpha_{1-1}}+y^{\alpha_{2-1}}\right) e^{-\left(\frac{x}{\beta_{1}}+\frac{y}{\beta_{2}}\right)} \\
& , x>0 ; y>0
\end{aligned}
$$

Then the nth moment of the joint distribution of $X^{c}$ and $Y^{d}$ about zero is

$$
\begin{aligned}
& \mu_{n}(c, d) \\
& =\frac{1}{\beta_{2} \beta_{1}^{\alpha_{1}} \sqrt{\alpha_{1}}+\beta_{1} \beta_{2}^{\alpha_{2}} \sqrt{\alpha_{2}}} \int_{0}^{\infty} \int_{0}^{\infty} x^{c n} y^{d n}\left(x^{\alpha_{1-1}}+y^{\alpha_{2-1}}\right) e^{-\left(\frac{x}{\beta_{1}}+\frac{y}{\beta_{2}}\right)} d x d y \\
& =\frac{1}{\beta_{2} \beta_{1}^{\alpha_{1}} \sqrt{\alpha_{1}}+\beta_{1} \beta_{2}^{\alpha_{2}} \sqrt{\alpha_{2}}} \int_{0}^{\infty} \int_{0}^{\infty} x^{c n} y^{d n} x^{\alpha_{1-1}} e^{-\left(\frac{x}{\beta_{1}}+\frac{y}{\beta_{2}}\right)} d x d y+ \\
& \frac{1}{\beta_{2} \beta_{1}^{\alpha_{1}} \sqrt{\alpha_{1}}+\beta_{1} \beta_{2}^{\alpha_{2}} \sqrt{\alpha_{2}}} \int_{0}^{\infty} \int_{0}^{\infty} x^{c n} y^{d n} y^{\alpha_{2-1}} e^{-\left(\frac{x}{\beta_{1}}+\frac{y}{\beta_{2}}\right)} d x d y \\
& \text { let } u=\frac{x}{\beta_{1}} \text { and } v=\frac{y}{\beta_{2}} \text { then } \\
& \left.\mu_{n}(c, d)=\frac{1}{\beta_{2} \beta_{1}^{\alpha_{1}} \sqrt{\alpha_{1}}+\beta_{1} \beta_{2}^{\alpha_{2}} \sqrt{\alpha_{2}}} \beta_{1}^{c n+\alpha_{1}} \beta_{2}^{d n+1} \sqrt{c n}+\alpha_{1}\right) \sqrt[d n]{ }+1+ \\
& \frac { 1 } { \beta _ { 2 } \beta _ { 1 } ^ { \alpha _ { 1 } } \sqrt { \alpha _ { 1 } } + \beta _ { 1 } \beta _ { 2 } ^ { \alpha _ { 2 } } \sqrt { \alpha _ { 2 } } } \beta _ { 1 } ^ { c n + 1 } \beta _ { 2 } ^ { d n + \alpha _ { 2 } } \sqrt { c n } + 1 \longdiv { d n } + \alpha _ { 2 } .
\end{aligned}
$$

Hence

$$
\mu_{n}(c, d)=\frac{\left.\beta_{1}^{c n+\alpha_{1}} \beta_{2}^{c n+1} \sqrt{c n}+\alpha_{1} \sqrt{d n}+1+\beta_{1}^{c n+1} \beta_{2}^{d n+\alpha_{2}} \sqrt{c n}+1\right) \sqrt{d n}+\alpha_{2}}{\beta_{2} \beta_{1}^{\alpha_{1}} \sqrt{\alpha_{1}}+\beta_{1} \beta_{2}^{\alpha_{2}} \sqrt{\alpha_{2}}}
$$

Then the nth moment of the marginal distribution of $X^{c}$ about zero is obtained by setting $\mathrm{d}=0$ in Equation 16 , to give

$$
\begin{aligned}
& \mu_{n}(c)=\mu_{n}(c, 0) \\
& =\frac{\left.\beta_{2} \beta_{1}^{c n+\alpha_{1}} \sqrt{c n}+\alpha_{1} \beta_{1}^{c n+1} \beta_{2}^{\alpha_{2}} \sqrt{c n}+1\right) \alpha_{2}}{\beta_{2} \beta_{1}^{\alpha_{1}} \sqrt{\alpha_{1}}+\beta_{1} \beta_{2}^{\alpha_{2}} \sqrt{\alpha_{2}}}
\end{aligned}
$$

Similarly the nth moment of the marginal distribution of $Y^{d}$ about zero is obtained by setting $\mathrm{c}=0$ in Equation 16 to obtain

$$
\begin{aligned}
& \mu_{n}(d)=\mu_{n}(0, d) \\
& =\frac{\beta_{1}^{\alpha_{1}} \beta_{2}^{d n+1} \sqrt{\alpha_{1}} \cdot \sqrt{d n}+1+\beta_{1} \beta_{2}^{d n+\alpha_{2}} \sqrt{d n}+\alpha_{2}}{\left.\beta_{2} \beta_{1}^{\alpha_{1}}\right) \alpha_{1}}+\beta_{1} \beta_{2}^{\alpha_{2}} \sqrt{\alpha_{2}}
\end{aligned}
$$

Note that if in Equation 15 we set $\alpha_{1}=\frac{k_{1}}{2}, \alpha_{2}=\frac{k_{2}}{2}$ and $\beta_{1}=\beta_{2}=2$,we have a bi-variate chisquare distribution then the corresponding nth moment of the joint distribution of $X^{c}$ and $Y^{d}$ is obtained by setting $\alpha_{1}=\frac{k_{1}}{2}, \alpha_{2}=\frac{k_{2}}{2}$ and $\beta_{1}=\beta_{2}=2$ in Equation 16 which yields,

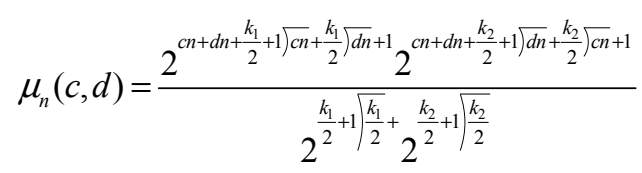

or

$$
=\frac{2^{c n+d n+1}\left(2^{\frac{k_{1}}{2}} \sqrt{c n}+\frac{k_{1}}{2} \sqrt{d n}+1+2^{\frac{k_{2}}{2}} \sqrt{d n}+\frac{k_{2}}{2} \sqrt{c n}+1\right)}{2^{\frac{k_{1}}{2}+1 \frac{k_{1}}{2}+} 2^{\frac{k_{2}}{2}+1 / \frac{k_{2}}{2}}}
$$

The nth moment of the marginal distribution of $X^{c}$ given this bi-variate chi-square distribution is obtained by setting $\mathrm{d}=0$ in Equation 19 yielding

$$
{ }_{x} \mu_{n}(c)=\mu_{n}(c, 0)=\frac{2^{c n+1}\left(2^{\frac{k_{1}}{2}} \sqrt{c n}+\frac{k_{1}}{2}+2^{\frac{k_{2}}{2}} \frac{k_{2}}{2} \sqrt{c n}+1\right)}{2^{\frac{k_{1}}{2}+1 \frac{k_{1}}{2}+\frac{k_{2}}{2}+1 / \frac{k_{2}}{2}} 2^{\frac{k^{2}}{2}}}
$$

The corresponding nth moment of the marginal distribution of $Y^{d}$ about zero is similarly obtained by setting $\mathrm{c}=0$ in Equation 19.If in Equation 15,we set $\alpha_{1}=\alpha_{2}=1$,we have the bi-variate exponential distribution. We the obtain the corresponding nth moment of the joint distribution of $X^{c}$ and $Y^{d}$ given this bi-variate exponential distribution by setting $\alpha_{1}=\alpha_{2}=1$ in Equation 16 as

$$
\begin{aligned}
& \mu_{n}(c, d)=\frac{\left.\beta_{1} \beta_{2} \beta_{1}^{c n+1} \beta_{2}^{d n} \sqrt{c n}+1 \sqrt{d n}+1+\beta_{1} \beta_{2} \beta_{1}^{c n} \beta_{2}^{d n+1} \sqrt{c n}+1\right) \sqrt{d n}+1}{2 \beta_{1} \beta_{2}} \\
& =\frac{\beta_{1} \beta_{2} \beta_{1}^{c n} \beta_{2}^{d n} \sqrt{c n}+1 \sqrt{d n}+1+\beta_{1} \beta_{2} \beta_{1}^{c n} \beta_{2}^{d n} \sqrt[c n]{c n} 1 \sqrt{d n}+1}{2 \beta_{1} \beta_{2}} \\
& \mu_{n}(c, d)=\beta_{1}^{c n} \beta_{2}^{d n} \sqrt{c n}+1 \sqrt[d n]{d n}+1
\end{aligned}
$$

The nth moment of the marginal distribution of $X^{c}$ about zero, given this joint exponential distribution is obtained by setting $\mathrm{d}=0$ in Equation 21 and is given as

$$
\mu_{n}(c, 0)=\beta_{1}^{c n} \overline{c n}+1
$$

Note that if in Equation 18, we set $\mathrm{c}=\mathrm{d}=1$, we have the nth moment of the bi-variate Gamma distribution of Equation 15 about zero, which is

$$
\begin{aligned}
& \mu_{n}(1,1)=\frac{\beta_{2} \beta_{1}^{n+\alpha_{1}} \beta_{2}^{n} \sqrt{n}+\alpha_{1} \sqrt{n}+1+\beta_{1} \beta_{1}^{n} \beta_{2}^{n+\alpha_{2}} \sqrt{n}+1 \sqrt{n}+\alpha_{2}}{\beta_{2} \beta_{1}^{\alpha_{1}} \sqrt{\alpha_{1}}+\beta_{1} \beta_{2}^{\alpha_{2}} \sqrt{\alpha_{2}}} \\
& =\frac{\beta_{2} \beta_{1}^{\alpha_{1}} \beta_{1}^{n} \beta_{2}^{n} \sqrt{n}+\alpha_{1} \sqrt{n}+1+\beta_{1} \beta_{2}^{\alpha_{2}} \beta_{1}^{n} \beta_{2}^{n} \sqrt{n}+\alpha_{2} \sqrt{n}+1}{\beta_{2} \beta_{1}^{\alpha_{1}} \sqrt{\alpha_{1}}+\beta_{1} \beta_{2}^{\alpha_{2}} \sqrt{\alpha_{2}}}
\end{aligned}
$$

that is

$$
\mu_{n}(1,1)=\frac{\beta_{1}^{n+\alpha_{1}} \beta_{2}^{n+1} \sqrt{n}+\alpha_{1} \sqrt{n}+1+\beta_{1}^{n+1} \beta_{2}^{n+\alpha_{2}} \sqrt{n}+\alpha_{2} \sqrt{n}+1}{\beta_{2} \beta_{1}^{\alpha_{1}} \sqrt{\alpha_{1}}+\beta_{1} \beta_{2}^{\alpha_{2}} \sqrt{\alpha_{2}}}
$$


The corresponding nth moment of the bi-variate chisquared distribution about zero is obtained by setting $\mathrm{c}=\mathrm{d}=1$ in Equation 19 yielding

$$
\mu_{n}(1,1)=\frac{\left.2^{2 n+1}\left(2^{\frac{k_{1}}{2}} \sqrt{n}+\frac{k_{1}}{2} \sqrt{n}+1+2^{\frac{k_{2}}{2}} \sqrt{n}+\frac{k_{2}}{2}\right) n+1\right)}{2^{\frac{k_{1}}{2}+1 \frac{k_{1}}{2}+} 2^{\frac{k_{2}}{2}+1 / \sqrt[k_{2}]{2}}}
$$

The nth moment of the marginal distribution of $\mathrm{X}$ (which is now chi-squared distributed) about zero is obtained as

$$
\begin{aligned}
& { }_{x} \mu_{n}(c)=\mu_{n}(c, 0) \\
& =\frac{2^{2 n+1}\left(2^{\frac{k_{1}}{2}} \sqrt{n}+\frac{k_{1}}{2}+2^{\frac{k_{2}}{2}} \sqrt{\frac{k_{2}}{2}} \cdot \sqrt{n}+1\right)}{2^{\left.\frac{k_{1}}{2}+1\right) \frac{k_{1}}{2}}+2^{\left.\frac{k_{2}}{2}+1\right) \frac{k_{2}}{2}}}
\end{aligned}
$$

Also, setting $\mathrm{c}=\mathrm{d}=1$ in Equation 21 we obtain the nth moment of the bi-variate exponential distribution about zero as

$$
\mu_{n}(1,1)=\beta_{1}^{n} \beta_{2}^{n}(\sqrt{c n}+1)^{2}
$$

The corresponding nth moment of the marginal distribution of $\mathrm{X}$ (which is now exponentially distributed) about zero is obtained by setting $\mathrm{d}=0$ in Equation 21 which yields

$$
{ }_{x} \mu_{n}(1)=\mu_{n}(1,0)=\beta_{1}^{n} \sqrt{n}+1
$$

The mgf of the bi-variate Gamma distribution of Equation 25 is easily obtained as

$$
\begin{aligned}
& M\left(t_{1}, t_{2}\right) \\
& =\frac{\beta_{2}\left(1-\beta_{2} t_{2}\right)^{-1} \beta_{1}^{\alpha_{1}}\left(1-\beta_{1} t_{1}\right)^{-\alpha_{1}} \sqrt{\alpha_{1}}+\beta_{1}\left(1-\beta_{1} t_{1}\right)^{-1} \beta_{2}^{\alpha_{1}}\left(1-\beta_{2} t_{2}\right)^{-\alpha_{2}} \sqrt[\alpha_{2}]{\beta_{2} \beta_{1}^{\alpha_{1}} \sqrt{\alpha_{1}}+\beta_{1} \beta_{2}^{\alpha_{2}} \sqrt{\alpha_{2}}}}{}
\end{aligned}
$$

Equation 23 is easier and quicker to use in finding the nth moment of the bi-variate Gamma distribution of Equation 15 than differentiating $\mathrm{n}$ times the corresponding mgf given in Equation 28 with respect $t_{0}, t_{1}$ and $t_{2}$ evaluating the result at $t_{1}=t_{2}=0$. Similarly, the Equations for the nth moment of the indicated marginal distributions are easier and quicker to use than the corresponding marginal mgf in finding these moments. To illustrate further, if $\alpha_{1}=\alpha_{2}=2$,we have the pdf

$$
\begin{aligned}
f(x, y) & =\frac{(x+y) e^{-\left(\frac{x}{\beta_{1}}+\frac{y}{\beta_{2}}\right)}}{\beta_{1} \beta_{2}\left(\beta_{1}+\beta_{2}\right)} \\
& , x>0, y>0
\end{aligned}
$$

Suppose $\mathrm{X}$ and $\mathrm{Y}$ have the bi-variate Releigh distribution with pdf

$$
\begin{aligned}
& f(x, y)= \\
& =\frac{4 \alpha_{1} \alpha_{2} \sqrt{\alpha_{1} \alpha_{2}}}{\sqrt{\pi}\left(\alpha_{1} \sqrt{\alpha_{2}}+\alpha_{2} \sqrt{\alpha_{1}}\right)}(x+y) e^{-\left(\alpha_{1} x^{2}+\alpha_{2} y^{2}\right)}, x>0, y>0
\end{aligned}
$$

\section{then $\mu_{n}(c, d)$}

$\mu_{n}(c, d)=$

$$
\begin{aligned}
& =\frac{4 \alpha_{1} \alpha_{2} \sqrt{\alpha_{1} \alpha_{2}}}{\sqrt{\pi}\left(\alpha_{1} \sqrt{\alpha_{2}}+\alpha_{2} \sqrt{\alpha_{1}}\right)} \int_{0}^{\infty} \int_{0}^{\infty} x^{c_{n}} y^{d n}(x+y) e^{-\left(\alpha_{1} x^{2}+\alpha_{2} y^{2}\right)} d x d y \\
& =\frac{4 \alpha_{1} \alpha_{2} \sqrt{\alpha_{1} \alpha_{2}}}{\sqrt{\pi}\left(\alpha_{1} \sqrt{\alpha_{2}}+\alpha_{2} \sqrt{\alpha_{1}}\right)}\left(x^{c n+1} y^{d n} e^{-\left(\alpha_{1} x^{2}+\alpha_{2} y^{2}\right)} d x d y+x^{a n} y^{d n+1} e^{-\left(\alpha_{1} x^{2}+\alpha_{2} y^{2}\right)} d x d y\right)
\end{aligned}
$$

let $u=\alpha_{1} x^{2}$ and $v=\alpha_{2} y^{2}$

then the $u=2 \alpha_{1} x d x$ and the $v=2 \alpha_{2} y d y$.

$\therefore \mu_{n}(c, d)=$

$=\frac{\alpha_{1} \alpha_{2} \sqrt{\alpha_{1} \alpha_{2}}}{\sqrt{\pi}\left(\alpha_{1} \sqrt{\alpha_{2}}+\alpha_{2} \sqrt{\alpha_{1}}\right)}\left(x^{g n+1} y^{g n} e^{-\left(\alpha_{1} x^{2}+\alpha_{2} y^{2}\right)} d x d y+x^{g n} y^{g h+1} e^{-\left(\alpha_{1} x^{2}+\alpha_{2} y^{2}\right)} d x d y\right)$

$\mu_{n}(c, d)=$

$=\left(\alpha_{1}^{-\left(\frac{n}{2}+1\right)} \alpha_{2}^{-\left(\frac{d n}{2}+1\right)} \int_{0}^{\infty} \int_{0}^{\infty} u^{\frac{c}{2}} v^{\frac{d n}{2}-\frac{1}{2}} d u d v+\alpha_{2}^{-\left(\frac{m}{2}+1\right)} \alpha_{1}^{-\left(\frac{n}{2}+1\right)} \int_{0}^{\infty} \int_{0}^{\infty} v^{\frac{d n}{2}} u^{\frac{c n}{2}-\frac{1}{2}} d u d v\right)$

therefore,

$$
\begin{aligned}
& \mu_{n}(c, d)=\frac{\alpha_{1} \alpha_{2} \sqrt{\alpha_{1} \alpha_{2}}}{\sqrt{\pi}\left(\alpha_{1} \sqrt{\alpha_{2}}+\alpha_{2} \sqrt{\alpha_{1}}\right)} \\
& \left.\left.\times\left(\alpha_{1}^{-\left(\frac{c n}{2}+1\right)} \alpha_{2}^{-\left(\frac{d n}{2}+1\right)} \sqrt{\frac{c n}{2}}+1\right) \sqrt{\frac{d n}{2}+1}+\alpha_{2}^{-\left(\frac{d n}{2}+1\right)} \alpha_{1}^{-\left(\frac{c n}{2}+1\right)} \sqrt{\frac{d n}{2}}+1\right) \sqrt{\frac{c n+1}{2}}\right)
\end{aligned}
$$

the moment of the marginal distribution of $X^{c}$ about zero is obtained by setting $\mathrm{d}=0$ in Equation 30 that is

$$
\begin{aligned}
& { }_{x} \mu_{n}(c)=\mu_{n}(c, 0)=\frac{\alpha_{1} \alpha_{2} \sqrt{\alpha_{1} \alpha_{2}}}{\sqrt{\pi}\left(\alpha_{1} \sqrt{\alpha_{2}}+\alpha_{2} \sqrt{\alpha_{1}}\right)} \\
& \left.\left.\times\left(\frac{\sqrt{\pi}}{\alpha_{2}} \alpha_{1}^{-\left(\frac{c n}{2}+1\right)}\right) \sqrt{\frac{c n}{2}}+1+\alpha_{2}^{-1} \alpha_{1}^{-\left(\frac{c n}{2}+1\right)}\right) \sqrt{\frac{c n}{2}}+1\right)
\end{aligned}
$$

If we set $c=d=1$,in Equation 30 we obtain the $n$th of the bi-variate Releigh distributions of Equation 29 about zero as

$$
\begin{aligned}
& \mu_{n}(1,1)=\frac{\alpha_{1} \alpha_{2} \sqrt{\alpha_{1} \alpha_{2}}}{\sqrt{\pi}\left(\alpha_{1} \sqrt{\alpha_{2}}+\alpha_{2} \sqrt{\alpha_{1}}\right)} \\
& \times\left(\alpha_{1}^{-\left(\frac{t}{2}+1\right)} \alpha_{2}^{-\left(\frac{t}{2}+1\right)} \sqrt{\frac{t}{2}}+1 \sqrt{\frac{t}{2}+1}+\alpha_{2}^{-\left(\frac{t}{2}+1\right)} \alpha_{1}^{-\left(\frac{t}{2}+1\right)} \sqrt{\frac{t}{2}}+1 \sqrt{\frac{t}{2}+1}\right)
\end{aligned}
$$

that is 


$$
\begin{aligned}
& \mu_{n}(1,1)=\frac{\alpha_{1} \alpha_{2} \sqrt{\alpha_{1} \alpha_{2}}}{\sqrt{\pi}\left(\alpha_{1} \sqrt{\alpha_{2}}+\alpha_{2} \sqrt{\alpha_{1}}\right)} \\
& \times\left(\sqrt{\frac{t}{2}}+1 \sqrt{\frac{t}{2}+1}\left(\alpha_{1}^{-\left(\frac{t}{2}+1\right)} \alpha_{2}^{-\left(\frac{t}{2}+1\right)}+\alpha_{2}^{-\left(\frac{t}{2}+1\right)} \alpha_{1}^{-\left(\frac{t}{2}+1\right)}\right)\right)
\end{aligned}
$$

The marginal of $\mathrm{X}$ is obtained from Equation 30 by setting $\mathrm{c}=1$ and $\mathrm{d}=0$ in Equation 30 as

$$
\begin{aligned}
& { }_{x} \mu_{n}(1)=\mu_{n}(1,0)=\frac{\alpha_{1} \alpha_{2} \sqrt{\alpha_{1} \alpha_{2}}}{\sqrt{\pi}\left(\alpha_{1} \sqrt{\alpha_{2}}+\alpha_{2} \sqrt{\alpha_{1}}\right)} \sqrt{\frac{\pi}{\alpha_{2}}} \\
& \times \alpha_{1}^{\left(\frac{t}{2}+1\right)} \sqrt{\frac{t}{2}}+1+\alpha_{2}^{-1} \alpha_{1}^{-\left(\frac{t}{2}+1\right)} \sqrt{\frac{t}{2}}+1
\end{aligned}
$$

Moment of continuous bi-variate distribution for normal Suppose $\mathrm{X}$ and $\mathrm{Y}$ have the bi-variate normal distribution with pdf

$$
\begin{aligned}
& f(x, y)=\frac{1}{2 \sigma_{1} \sigma_{2} \pi} e^{-\frac{1}{2}\left[\left(\frac{x-\mu_{1}}{\sigma_{1}}\right)^{2}+\left(\frac{y-\mu_{2}}{\sigma_{2}}\right)^{2}\right]}, \\
& \text { for }-\infty<x<\infty,-\infty<y<\infty,-\infty<\mu_{1}<\infty, \\
& -\infty<\mu_{2}<\infty, \sigma_{1}^{2}>0 ; \sigma_{2}^{2}>0 .
\end{aligned}
$$

Interest is to find the bivariate distribution of the joint distribution of the random variables $X^{c}$ and $Y^{d}$ where c and $\mathrm{d}$ are any real numbers. Therefore the nth moment of the joint distribution of $X^{c} Y^{d}$ about zero is

$$
\begin{aligned}
& \mu_{n}(c, d)^{\prime}= \\
& =\frac{1}{2 \sigma_{1} \sigma_{2} \pi} \int_{-\infty}^{\infty} \int_{-\infty}^{\infty} x^{c n} y^{d n} e^{-\left[\left(\frac{x-\mu_{1}}{\sigma_{1} \sqrt{2}}\right)^{2}+\left(\frac{y-\mu_{2}}{\sigma_{2} \sqrt{2}}\right)^{2}\right]} d x d y \\
& \text { Let } u=\left(\frac{x-\mu_{1}}{\sigma_{1} \sqrt{2}}\right)^{2} \text { and } v=\left(\frac{y-\mu_{2}}{\sigma_{2} \sqrt{2}}\right)^{2},
\end{aligned}
$$

Integrating and substituting, we have

$$
\mu_{n}(c, d)^{\prime}=\frac{1}{\pi} \int_{0}^{\infty} \int_{0}^{\infty}\left(\sigma_{1} \sqrt{2} \cdot u^{\frac{1}{2}}+\mu_{1}\right)^{c n} u^{-\frac{1}{2}} \cdot\left(\sigma_{2} \sqrt{2} \cdot v^{\frac{1}{2}}+\mu_{2}\right)^{d n} \cdot v^{-\frac{1}{2}} e^{-(u+v)} d u d v
$$

Using binomial expansion, we have

$$
\mu_{n}(c, d)^{\prime}=\frac{1}{\pi} \sum_{t=0}^{n}\left(\begin{array}{c}
c n \\
t
\end{array}\right) \mu_{1}^{c n-t}\left(2 \sigma_{1}^{2}\right)^{\frac{t}{2}} \int_{0}^{\infty} u^{\frac{1}{2}+\frac{1}{2}-1} e^{-u} d u \sum_{s=0}^{d n}\left(\begin{array}{c}
d n \\
s
\end{array}\right) u_{2}^{u n-s}\left(2 \sigma_{2}^{2}\right)^{\frac{s}{2}} \int_{0}^{\infty} v^{\frac{s}{2}+\frac{1}{2}-1} e^{-v} d v
$$

That is,

$$
\begin{aligned}
& \mu_{n}(c, d)^{\prime}= \\
& =\sum_{t=0}^{c n}\left(\begin{array}{c}
c n \\
t
\end{array}\right) \mu_{1}^{c n-t}\left(2 \sigma_{1}^{2}\right)^{\frac{t}{2}} \frac{\frac{\sqrt{t}}{2}+\frac{1}{2}}{\sqrt{\pi}} \sum_{s=0}^{d n}\left(\begin{array}{c}
d n \\
s
\end{array}\right) \mu_{2}^{d n-s}\left(2 \sigma_{2}^{2}\right)^{\frac{s}{2}} \frac{\frac{\sqrt{s}}{2}+\frac{1}{2}}{\sqrt{\pi}}
\end{aligned}
$$

For $\mathrm{t}, \mathrm{s}=0,2 \ldots \ldots$, that is provided $\mathrm{t}$ and $\mathrm{s}$ are even numbers. In other words provided we set

$$
\left(2 \sigma_{1}^{2}\right)^{\frac{t}{2}} \frac{\frac{\sqrt{t}}{2}+\frac{1}{2}}{\sqrt{\pi}}=\left(2 \sigma_{2}^{2}\right)^{\frac{s}{2}} \frac{\frac{\sqrt{s}}{2}+\frac{1}{2}}{\sqrt{\pi}}=0
$$

For all odds values of $\mathrm{t}$ and $\mathrm{s}$ since for example with $v=\frac{x-\mu}{\sigma}$, we have that $\frac{1}{2 \sqrt{\pi}} \int_{-\infty}^{\infty} v^{t} \cdot e^{-\frac{1}{2} \cdot \frac{v}{2}} d v=0$, for all odds values of ' $t$ ' that is for $t=1,3,5, \ldots \ldots$ as may easily be verifed.

\section{Summary and Conclusion}

We have presented in this paper method of obtaining the moment of some continuous bi-variate distributions with parameters $\alpha_{1}, \beta_{1}$ and $\alpha_{2}, \beta_{2}$ in finding the $n$th moment of the variable $x^{c} y^{d}(c \geq 0, d \geq 0)$ where $\mathrm{X}$ and $\mathrm{Y}$ are continuous random variables having the joint pdf, $\mathrm{f}(\mathrm{x}, \mathrm{y})$.The proposed methods were the so called $g_{n}(c, d)$ defined $g_{n}(c, d)=E\left(X^{c} Y^{d}+\lambda\right)^{n}$, the nth moment of expected value of the $t$ distribution of the cth power of $X$ and $d$ th power of $\mathrm{Y}$ about the constant $\lambda$. These moments are obtained by the use of bi-variate moment generating functions, when they exist. The proposed $g_{n}(c, d)$ exists for all continuous probability distributions unlike some of its competitors such as factorial moments of moment generating function which do not always exist. The results obtained using $g_{n}(c, d)$ are the same as results obtained using such other methods as moment generating functions of available. The proposed method is available and easy to use without the need for any modifications even when the powers of the random variable being considered are nonnegative real numbers that do not need to be integers. The results obtained using $g_{n}(c, d)$ are the same as results obtained using other methods such as moment generating functions when they exist.

\section{References}

[1] Baisnab AP, Manoranjan J (1993). Elements of Probability and Statistics, Tata McGraw - Hill Publishing Company Limited, New Delhi. pp. 208-232.

[2] Freund JE (1992). Mathematical Statistics (5th Edition), Prentice - Hall International Editions, USA. pp. 161-177.

[3] Hay, William (1973).Statistics for the social sciences: Holt, Rinchart and Winston Inc New York, PP 778-780.

[4] Spiegel M.R (1998): Theory and Problems of Statistics, McGraw-Hill Book Company, New York. Pp 261-264.

[5] Uche PI (2003). Probability: Theory and Applications, Longman Nigeria PLC, Ikeja Lagos. pp. 149-155. 